\title{
Les Ressources en eaux dans la Plaine du Moyen Cheliff*
}

\section{Zerarka Souad**}

\begin{abstract}
RÉSUMÉ
Le nord-ouest de L'Algérie est menacé par la sécheresse et la rareté de l'eau attribue pour l'irrigation; a cause de l'extension urbaine qui a engendré une demande accrue de l'eau à des usagers différents (alimentation en eau potable; industrie; et irrigation. Les plaines de Cheliff sont en outre considérées comme les plus fertiles des plaines intra-telliennes de l'Algérie. Dès 1848, elles furent colonisées par les Français et différents ouvrages hydrauliques y furent réalisés. Dans sa thèse de Doctorat «La colonisation des plaines du Cheliff» 1955, Xavier Yacono fait état de différentes infrastructures qui en fait, depuis, des périmètre irrigués les plus fertiles d'Algérie: barrage d'Oum Drou (1872), Barrage d'Oued Fodda (1932), canaux d'irrigation reliant ces barrages aux périmètres irrigués produisant blé, agrumes et raisin.

Ces plaines s'étendent d'Est en Ouest sur une surface de $300 \mathrm{~km}$ et sont traversées par le plus long oued d'Algérie, l'Oued Cheliff long de 750km qui constitue le pôle économique le plus attractif de la région.

Ces plaines sont caractérisées par un climat rude et contrasté avec des étés torrides et des hivers très froids malgré leur situation à seulement $40 \mathrm{~km}$ de la mer (barrière de la montagne du Dahra), cette particularité leur a valu des qualitatifs célèbres tels: «Four du Tell» ou «Portion du Sahara égarée au Tell» Xavier Yacono» (Yacono-Boulaine).

L'Oued Cheliff, alimenté par un dense réseau hydrographique en constitue la l'ossature principale et l'amont de son activité humaine et économique.

Cependant l'irrégularité saisonnière et interannuelle de l'écoulement des intempéries, accentuée par de longues périodes de sécheresse, des crues violentes et brèves, une érosion intense avec des transports solides importants, un taux d'envasement considérable des barrages en exploitations (54.9\%), un taux de remplissage irrégulier de 37.51 à $72.42 \%$ au cours de l'année 2011-2016; sont autant de facteur qui ont engendré une réduction des superficies irriguées. N'oublions pas également les problèmes posés par la mauvaise gestion de l'eau auxquels il faut ajouter la vétusté des réseaux d'irrigation, la tarification à revoir des eaux d'irrigation aggravée par le piquage illicite.

Autant de raisons qui ont déterminé le choix de notre article intitulé «Les ressources en eaux dans la plaine du moyen Cheliff», ce dernier fait partie de la plaine de Cheliff situé entre le haut Cheliff et le bas Cheliff. [Bul. Soc. Géog. d'Égypte, 2018, 91: 89-99]
\end{abstract}

Mots-clés: Hydraulique agricole, mobilisation des ressources en eau superficielles, bassin versant, mobilisation des ressources en eau Souterraines, étiage, méandre, sols hydro morphes.

* Encadrée par: Setti m'hammed, Professeur en Géographie, Université des sciences et de la technologie Houari Boumediene, Faculté des sciences de la terre et aménagement de territoire.

** Maitre-assistante, A: Géographe, Ecole Normale Superieure Bouzareah (ENS) ALGER, Algérie.

$-89-$ 


\section{Introduction}

Le moyen Cheliff est considéré parmi des plus anciennes plaines intérieures et occidentales les plus fertiles en Algérie, a été officiellement créé par le gouvernement français en 1936, il étend un rectangle $75 \mathrm{~km}$ et une superficie totale de 362600 hectares. Cette plaine côtière de la ville de Ténès est à environ $40 \mathrm{~km}$, mais c'est un bas fermé sur la mer, ce qui le rend sec et continental. La plaine coule d'est en ouest, le plus grand cours d'eau du nord de l'Algérie, la «vallée du Cheliff», soutenue par ses grands affluents, principale source d'eau d'irrigation dans la plaine. La plupart des activités économiques dépendent de l'agriculture. Ces solives exposées à des dans le volume d'eau en raison du manque de précipitations et des températures élevées, ont conduit à une contraction des exploités et irriguées zones agricoles, sont donc des centaines d'hectares de terres agricoles assoiffée et transformées cultures agricoles irriguées, la majeure partie des cultures pluviales (pluie), en plus de la présence des sols hydrau morphe résultant de l'ancien marais .sans compter expansion urbaine et les constructions illégales se déroulant facilement, est devenu un concours et menacent gravement la quantité d'eau destinée à l'irrigation moyen Cheliff.

\section{Etude des ressources en eau dans la plaine}

L'eau est un important facteur naturels qui contrôlent la production agricole où l'eau est disponible pour l'irrigation affecte la superficie des terres cultivées, et la structure des cultures et des taux de production agricole, Ces ressources varient selon l'origine, la taille et les formes de la nature, en fonction des conditions naturelles et climatiques de la région à laquelle appartient la zone d'étude. Les ressources naturelles de la plaine du moyen Cheliff sont divisées en deux types: ressources en eaux superficielles et eaux souterraines.

\section{Les ressources en eau de surface du Moyen Cheliff}

L'eau de surface est l'eau courante dans les rivières, les vallées temporaires, les pistes, les lacs frais, les océans et les mers, et la neige est le sommet le plus élevé de la montagne. L'eau de surface est la principale source d'eau souterraine renouvelable.

Selon les données recueillies par la Direction des ressources en eau de la wilaya de Chlef 2010, les eaux de surface de la wilaya étaient de 11,67 $\mathrm{hm}^{3}$. Cette «quantité insuffisante» d'eau est évacuée par de nombreux oueds provenant des petits bassins du bassin de Cheliff, reflétant la fluctuation et l'irrégularité de leur précipitation au cours de l'année. 


\section{Étude du réseau hydrographique du moyen Cheliff}

Le bassin de Cheliff s'étend entre $0^{\circ}$ et $30^{\circ} 30^{\prime}$ Est de Greenwich et $34^{\circ}$ et 36 30'nord de l'Equateur.il est classé par l'Agence Nationale des Ressources en en Eau, le plus grand bassin Collecteur d'Algérie, couvrant une superficie de $43750 \mathrm{~km}^{2}$ et constitué d'un réseau dense d'oueds et de tributaires.

Nous allons nous limiter à étudier les plus importants affluents passent par la plaine de Chéliff de la rive droite du oued jusqu'à la rive gauche de lui. Voir la carte ci-dessous.

\section{- Les affluents de la rive droite de oued Chellif :}

Il est un affluent de tiges principalement du côté ouest des montagnes du sud de Dahra et se caractérise par un manque d'eau reflète les petits bassins et le manque de précipitations en général, un flux temporaire et sec en été.

oued Ras: se compose d'un groupe d'affluents du côté ouest des montagnes Dahra terminé vallée au sud de Cheliff et la longueur de la tête d'une vallée $39 \mathrm{~km}$.

oued ouahrane: est un affluent de la oued de Cheliff, il recueille l'eau des montagnes et Dahra se jette dans la partie nord-ouest du bassin dans le cours d'eau de Cheliff, d'une longueur de $52 \mathrm{~km}$ et le débit annuel est de $4 \mathrm{Hm}^{3} / \mathrm{an}$.

\section{- Les affluents de la rive gauche de oued Chellif :}

La plupart de ses oueds proviennent des monts d'Ouarsenis, dont les affluents sont très différents des affluents de la rive droite et dont les principales caractéristiques sont l'écoulement continu de l'eau tout au long de l'année, la taille de ses bassins et sa présence face au vent, et d'absorber une grande partie de la pluie.

\section{Oued fodda :}

Ce oued provient des versants nord-est des monts ouarsnis et se caractérise par un chemin droit, sauf sur le côté nord il devient méandre. La longueur du oued fodda est de $75 \mathrm{~km}$ au barrage et de $117 \mathrm{~km}$ à l'estuaire, et son débit annuel est de $152,7 \mathrm{Hm}^{3}$ / an.

\section{Oued sly :}

Ses sources sont situées près des sources de la vallée de oued fodda, dans le sud-est du bassin près de Tesemselt, la longueur de la vallée est de $130 \mathrm{~km}$ et un débit annuel allant jusqu'à $111,3 \mathrm{hm}^{3} /$ ans. 

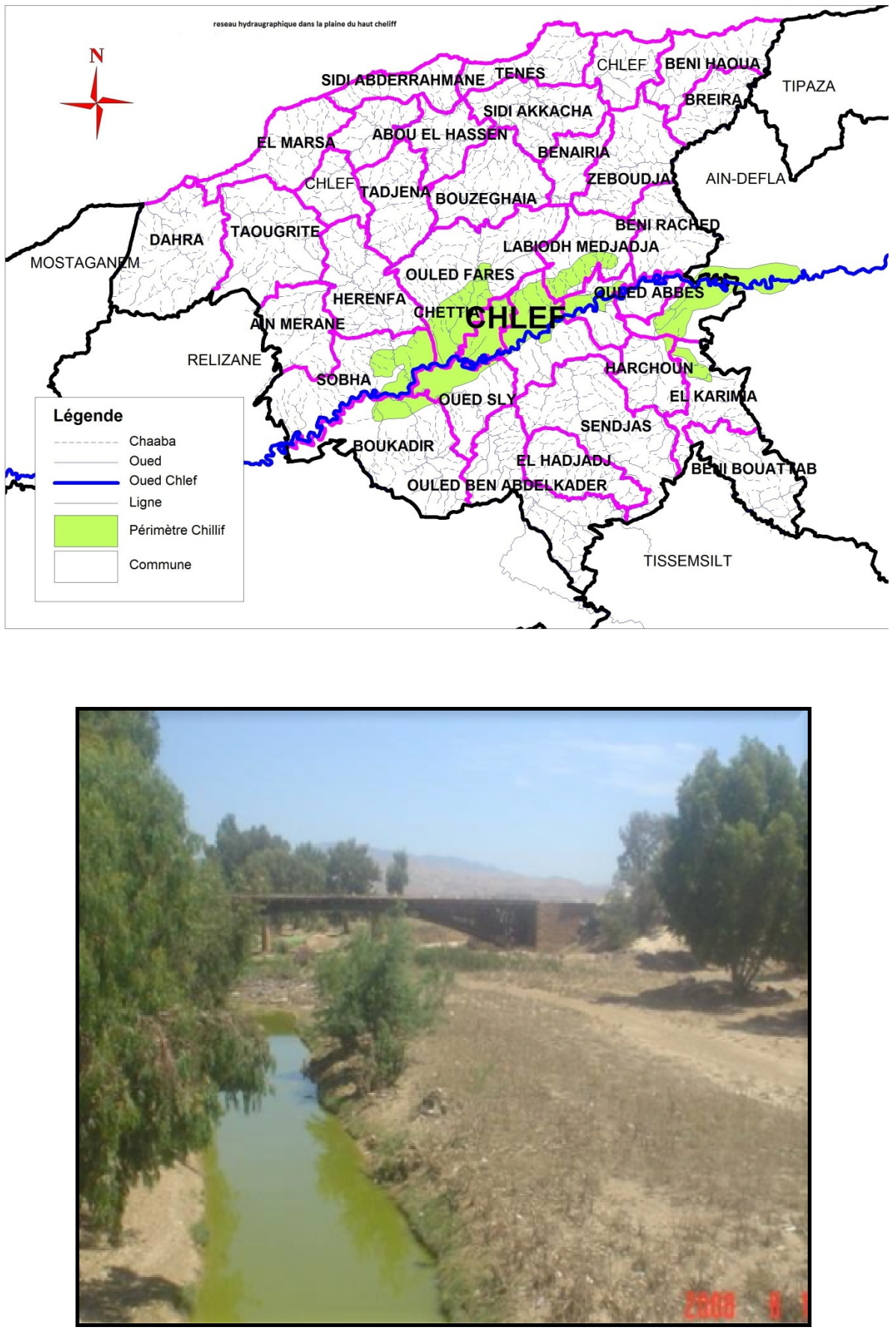

Photo 01. Photographie oued fodda en période d'étiage. 


\section{Oued tisighaout:}

Ce oued provient des versants nord des monts ouarsnis, en passant par la ville de Chlef, où elle se jette dans le cours principal de oues Chéliff.

Généralement, les oueds de la rive gauche de oued Chéliff sont des eaux de ruissellement dont les eaux s'assèchent en été, à l'exception du cours d'eau de oued fodda qui est permanent, mais à un niveau beaucoup plus bas qu'en hiver.

\section{Oued Cheliff:}

oued Cheliff, est le plus importante et le plus longue oued d'Algérie, c'est le seule oued septentrionales d'Algérie, originaire de l'atlas saharien et précisément des montagnes de l'Amour près de la ville d'Aflou qui se jette dans la mer au nord. Ce dernier coupe La plaine du moyen Cheliff d'est en ouest sur une longueur de $\mathbf{7 5} \mathbf{~ k m}$, formant une piste perpétuelle à faible dénivelé avec des précipitations annuelles moyennes de $393,9 \mathbf{h m}^{\mathbf{3}} / \mathbf{a n}$. Oued Cheliff est caractérisée par un système de drainage exoréique qui se jette dans la mer Méditerranée, à l'est de Mostaganem, et plus précisément de « Chélif plage».

De manière générale, la plupart des oueds d'Algérie «s'assèchent en été» en raison du faible rendement de leurs rendements à haute température et évaporation, attestée par la «sécheresse» ou étiage d'étiage, le plus grand cours d'eau d'Algérie, la vallée du Chéliff. Comme indiqué dans l'image cidessous.

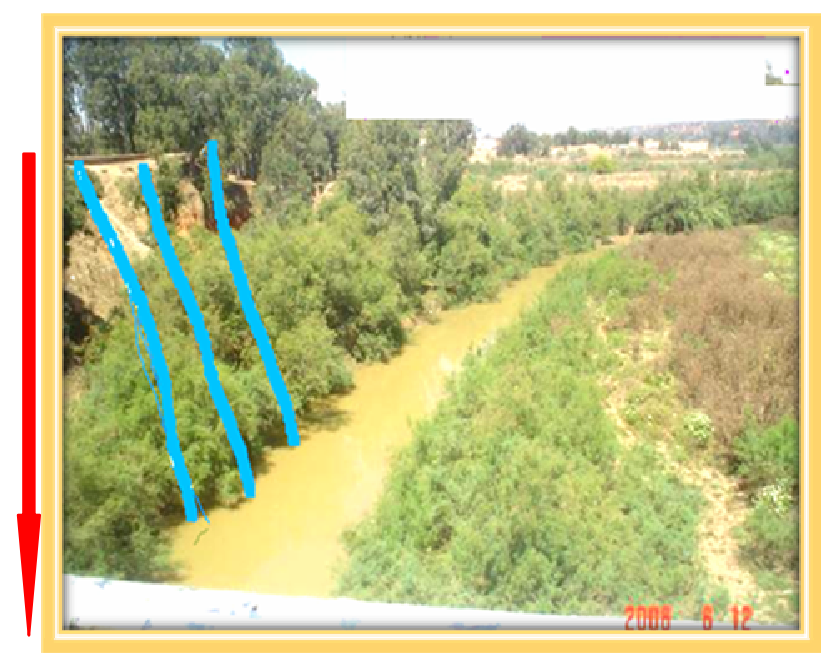

Photo 02: Le cours d'oued Cheliff passant par la ville de Chlef, en période. (étiage) Ceci est montré à travers le lit de l'oued. 
Mobilisation des eaux superficielles: sont mobilisées ceux qui stockent l'eau et massant dans des installations d'eau tels que les barrages et les retenues collinaires et des puits profonds et deux types de la normale, afin d'exploiter et utiliser au besoin. L'eau de pluie est considéré comme l'eau de surface des éléments nutritifs de base destiné à l' irrigation de vastes zones de la plaine, en mobilisant et emballés dans diverses structures et installations d'eau. Selon la direction des ressources en eau de la wilaya Chlef $10.49 \mathrm{hm}^{3}$ d'eau ont été mobilisés pour la création grands et petits barrages pour les exploiter dans les trois utilisations: l'eau potable, l'industrie et l'irrigation.

L'Algérie n'a pas connu une civilisation hydraulique (une civilisation basée sur les sources d'eau) sur le modèle ou l'image connue en Egypte ou en Mésopotamie, mais depuis l'antiquité apparue dans les communautés locales en plusieurs points de la patrie, elle est admirable Détournement des oueds dans les montagnes, arrosage des eaux de crue et étanchéité de l'eau pour utilisation).

Quant aux grands barrages, il a été associé à la naissance de l'ère coloniale, où le développement d'un grand programme de barrages en 1920.

L'achèvement des barrages a été un développement majeur de l'époque coloniale à nos jours De nombreuses structures d'eau ont été complétées pour recueillir l'eau de surface pour répondre aux besoins de la population dans divers domaines, agricoles, industriels et domestiques.

Selon la Direction des ressources en eau de la wilaya de Chlef en 2014, les ressources en eau de surface estimées à $10,49 \mathrm{Hm}^{3}$ ont été mobilisées par la construction de barrages et la construction des retenues collinaires principalement utilisées pour irrigation.

Les eaux de surface de la plaine du moyen Chéliff ont été modifiées par deux grands barrages d'une capacité supérieure à 10 millions de m3, exploités par l'Agence Nationale des Barrages et des Transferts (ANBT), dont l'exploitation et l'entretien sont assurés par oued fodda et oued Sidi Yacoub.

\section{Barrage de oued fodda}

Le barrage de oued fodda est le deuxième barrage achevé en Algérie après le «barrage de Beni Murad» en 1860. Le barrage de oued fodda est considéré comme l'un des plus grands barrages de la période coloniale. Il est situé à $20 \mathrm{~km}$ du village de oued fodda, exactement dans la municipalité de karimia . Il a été établi en 1932, avec une capacité de stockage de $228 \mathrm{Hm}^{3}$. 
Sa capacité régulière atteint $69 \mathrm{Hm}^{3}$ et est spécialement conçu pour irriguer la zone environnante du moyen Chéliff.

Barrage de Sidi Yacoub: Ce barrage est situé au nord du village Sidi Abdelkader, construit sur les gorges de la vallée de lardjem en 1985, affluent des principaux affluents de la rive gauche de oued chéliff, un barrage moderne destiné à approvisionner la wilaya de Chlef en eau potable, et pour irrigation du périmètre du bas Chéliff. La capacité de stockage du barrage est de $280 \mathrm{hm}^{3}$ et la capacité régulière est de $98 \mathrm{hm}^{3}$. Nous notons que l'énergie régulière est beaucoup plus faible que la capacité de stockage, comme le montre le tableau ci-dessous.

Tableau 01: Caractéristique et destination des barrages en Exploitation.

\begin{tabular}{|c|c|c|c|c|l|}
\hline $\begin{array}{c}\text { Nom du } \\
\text { barrage }\end{array}$ & $\begin{array}{c}\text { Nom } \\
\text { du } \\
\text { oued }\end{array}$ & $\begin{array}{c}\text { Date de } \\
\text { mise en } \\
\text { service } \\
\mathbf{H m}^{\mathbf{3}}\end{array}$ & $\begin{array}{c}\text { Volume } \\
\text { emmagasiné } \\
\mathbf{H m}^{\mathbf{3}}\end{array}$ & $\begin{array}{c}\text { Volume } \\
\text { régularisé }\end{array}$ & \multicolumn{1}{|c|}{ destination } \\
\hline $\begin{array}{c}\text { Oued } \\
\text { fodda }\end{array}$ & fodda & 1932 & 228 & 69 & $\begin{array}{l}\text { Irrigation du } \\
\text { moyen cheliff }\end{array}$ \\
\hline $\begin{array}{c}\text { Sidi } \\
\text { Ayoub }\end{array}$ & lardjem & 1985 & 280 & 98 & $\begin{array}{l}\text { Irrigation du } \\
\text { moyen et bas } \\
\text { cheliff, } \\
\text { alimentation de la } \\
\text { ville de chlef en } \\
\text { eau potable }\end{array}$ \\
\hline
\end{tabular}

Source: agence national des barrages et transfers, direction de maintenance et controle 20016.

Retenues collinaire: Ce sont de petits barrages d'une capacité de $50 \mathbf{H m}^{\mathbf{3}}$, réalisés par des moyens locaux situés dans la zone, dont le coût est relativement faible par rapport aux grands barrages que les propriétés demandent à construire sur leurs terres. À l'expertise étrangère dans leur achèvement.

La direction des ressources en eaux de la wilaya de Chlef supervise la gestion de ces petits barrages et les barrières suivantes pour répondre aux besoins croissants en eau, ce qui a entraîné l'expansion des zones agricoles irriguées.

Selon la Direction des resources en eau de la wilaya de Chlef, trois Retenues collinaires ont été dénombrées en 2014 dans la plaine du moyen Chlef, notamment la retenue collinaire suivante pour les de ouled Ben Abdelkader, et oum drou des, qui ont une capacité de $0,046 \mathrm{Hm}^{3}$ et 0,021

$-95-$ 
$\mathrm{Hm}^{3}$.respectivement, conviennent pour abreuver et boire les animaux, 100\% d'entre eux sont complètement inaptes à être utilisés, à part la retenue de Harchoune.

Petite barrage de Harchoun: Le barrage est situé dans la partie sud-est de la wilaya de Chlef dans la municipalité de harchoune, qui vise à fournir une source d'eau de 1,5 million de mètres cubes destiné à l'irrigation du périmetre du cheliff.

\section{Ressources en eaux souterraine}

L'eau souterraine est l'un des principaux éléments de l'eau de rivière permanente dans le monde, où le débit de base des rivières dépend des eaux souterraines. L'utilisation de l'eau souterraine augmente en augmentant le forage des puits, afin d'augmenter le besoin d'eau potable pour la population et d'eau d'irrigation pour l'agriculture dans de vastes régions du monde.

Les resources en eau souterraine de la wilaya de Chlef sont situées dans les strates composées principalement d'alluvions quaternaires sables astiens du Pliocene) et (calcaires à lithothamnium) qui sont des roches sédimentaires pouvant être conservées En grande quantité d'eau, appelée la couche contenant de l'eau L'aquifère, où le volume d'eau souterraine utilisé est d'environ $62 \mathbf{H m}^{3}$ / an, selon la Direction des ressources en eau de la wilaya de Chlef pour l'année 2014.

l'eau Mobilisés dans la wilaya de Chlef atteignait 25,08 $\mathbf{H m}^{3}$ selon la Direction des ressources en eaux de la wilaya de Chlef, où ont était mobilisée et exploitée dans la plaine du moyen Chéliff à partir de puits; et puits profonds et de sources.

Forages: ce sont des puits profonds; sont la principale ressource traditionnelle pour l'accès à l'eau souterraine dans les régions arides et semiarides du monde. Les puits profonds sont forés par des entreprises spécialisées, chaque puits profond atteignant quelques centaines de mètres et une profondeur de pas plus de 1000 mètres.

Selon l'Agence Nationale des Ressources en Eau et la Direction de l'Irrigation et de l'Agriculture de la wilaya de Chlef, 139 puits profonds d'une capacité de $1415344.4 \mathrm{~m}^{3}$ ont été distribués aux différentes municipalités de la plaine.

La quantité de puits profonds utilisée varie entre 3 et $301 / \mathrm{s}$ et est excavée à une profondeur de 90-214 $\mathrm{m}$ du sol, selon les sites aquifères souterrains situés dans la zone d'étude. 


\section{Puits traditionnels}

C'est une eau souterraine, qui est forée par les résidents dans l'œil du lieu, où la profondeur maximale est de $15 \mathrm{~m}$. La Direction de l'irrigation et de l'agriculture de l'Etat de Chlef compte 42 puits traditionnels d'une capacité de $140973 \mathrm{~m}^{3}$, pour la plupart privés, dont la consommation varie de 0,5 à 17 litres par hectare dans les différentes communes du moyen Chlef, pour alimenter en eau potable les habitants du moyen Cheliff et irriguer une partie de la plaine.

Sources: Les sources sont des ouvertures d'où l'eau coule à la surface sans pompage, pour apparaître à la surface de la terre sous la forme de ressorts et. Al-Ayoun était le principal fournisseur d'eau pour l'irrigation humaine et animale, et pour l'irrigation des fermes limitées dans les oasis

Et contiennent parfois certaines proportions d'éléments minéraux ou soufrés en plus de contenir d'autres minéraux utilisés dans les bains pour les loisirs et en tant que centre de guérison pour le traitement des patients de diverses maladies chroniques.

L'Agence Nationale des Ressources en Eau et la Direction de l'Irrigation de la wilaya de Chlef ont dénombré 13 sources répartis sur les différentes communes de la plaine d'une capacité de $49365 \mathrm{~m}^{3}$, tous exploités pour l'eau potable humaine et animale et l'irrigation des petites surfaces agricoles de la plaine.

Les eaux souterraines de la plaine du moyen Chéliff ont trois objectifs: fournir de l'eau potable aux habitants de la zone d'étude, irriguer les alentours et abreuver les animaux, le reste étant destiné à l'industrie.

\section{Conclusion}

Le moyen Cheliff est caractérisé par les ressources limitées en eau de surface malgré son potentiel hydrique et son emplacement dans le plus grand bassin de collecte du nord de l'Algérie, le bassin de Cheliff, pour des raisons climatiques et hydrologiques caractérisant cette zone d'étude.

Cependant, Les ressources souterraines dans la plaine sont considérées, et elles sont menacées par le risque de surexploitation. Suite à la mise en œuvre du plan national de développement agricole, les eaux souterraines ont été exploitées excessivement par certains agriculteurs à travers le creusement aléatoire de puits, de manière chaotique et illégale, et non en conformité avec les normes de 
forage de puits, L'exploitation de cet aquifère a appauvri l'aquifère, en l'absence de la police de l'eau préconisée par l'article 159, 160 de la loi sur l'eau, qui interdit et punit toute personne qui contrevient à cette loi.

Et à la lumière des enjeux sérieux que connaît le monde en raison des changements climatiques et de la sécheresse qui en découle, nous serons confrontés à l'obsession de la pénurie d'eau ou de la «pénurie d'eau» en 2025 Nous devrons ajouter de l'eau, dessalé et épuré pour combler le vide ou compenser la pénurie d'eau enregistrée dans la plaine du moyen Cheliff, en particulier que le secteur agricole dans la zone d'étude est le secteur le plus consommateur d'eau. Sachant que $82 \mathrm{hm}^{3}$ destiné à l'irrigation et $35,01 \mathrm{hm}^{3}$ en eau potable, tandis que l'industrie a reçu une petite prime, soit $0,56 \mathrm{hm}^{3}$.

\section{Bibliographie}

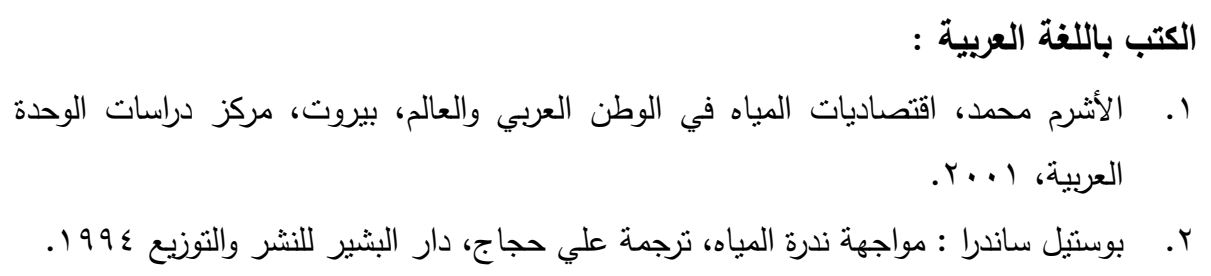

\section{Ouvrages en Français:}

1. Boulaine Jean: Géographie des sols, collection Sup, Paris, 1975.

2. Benamrane Djilali: Agriculture et développement en Algérie, Société nationale d'édition et de diffusion, Alger 1980.

3. Conac Françoise: Irrigation et développement agricole, éditionSedes réunis, Paris 1978.

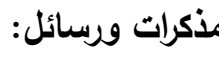

$$
\begin{aligned}
& \text { ا. العكري الحاج: النظام الهيدروغرافي لنهر الثلف، رسالة دكتوراه من الدور الثالث في الجغرافيا، } \\
& \text { معهد الجغرافيا، جامعة الجزائر } 19 V V . \\
& \text { r. ذوادي مهيدي: معوقات تكثيف الزراعة الجزائرية، رسالة ماجستير في العلوم الاقتصادية، فرع }
\end{aligned}
$$

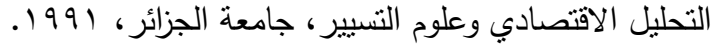




\section{Thèse et mémoires:}

1. Boulaine Jean, Etude des sols des plaines du Chéliff, thèse présentée pour obtention le grade de Docteur des Sciences Naturelles. Université d'Alger, Alger, 1957.

\section{Etude:}

1. Bacta M.S: Actes du séminaire Euro Méditerranéenne. Les instruments économiques et la modernisation des Périmètre irriguée, 21 \& 22 Novembre, Sousse, Tunisie 2005.

2. L'Aménagement du Territoire: L'Algérie de 2020, Les enjeux d'un développement durable.

3. Ministère des ressources en Eau, séminaire sur le secteur de L'Eau en Algérie (avec la collaboration de la Banque Mondiale). 